\title{
Arbeitnehmerbeteiligung in der Europäischen Aktiengesellschaft (SE) - Empirische Befunde und (un-)erwartete Konsequenzen
}

\author{
Berndt Keller \\ Frank Werner
}

Die Zahl der Unternehmen, die sich für die neue optionale Rechtsform der Europäischen Aktiengesellschaft (lat. Societas Europaea SE) entscheidet, wächst seit deren Einführung im Oktober 2004. Mit diesem deutlichen Anstieg gehen neue, vielfach unerwartete und auch vom Gesetzgeber nicht antizipierte Phänomene einher, besonders mit Blick auf die Arbeitnehmerbeteiligung. Der Beitrag leistet eine empirische Bestandsaufnahme (Stand Ende 2008), analysiert die Situation der SE, vor allem die verhandelte Arbeitnehmerbeteiligung, und zieht erste Schlussfolgerungen für die deutsche Mitbestimmungssituation und -diskussion.*

\section{1 \\ Einleitung}

\subsection{UNTERNEHMENSMIT- BESTIMMUNG - EIN STRITTIGES RECHTSINSTITUT}

Über die Zukunft der Mitbestimmung wird insbesondere auch vor dem Hintergrund einer zunehmenden Europäisierung kontrovers debattiert (Seyboth/Thannisch 2008). Mehrere Kommissionen (Bertelsmann-Stiftung/Hans-Böckler-Stiftung 1998; Biedenkopf et al. 2006) und Studien (Vitols 2006; Renaud 2007) kommen zu dem Ergebnis, dass sich die deutsche Mitbestimmung im Großen und Ganzen bewährt und keine negativen Auswirkungen auf den wirtschaftlichen Erfolg der Unternehmen hat, sondern im Gegenteil zu positiven Produktivitätseffekten führen kann. Trotz dieser empirischen Ergebnisse kritisieren bestimmte Interessengruppen wiederholt die geltenden Regelungen (BDA/ BDI 2004; Donges et al. 2007; Vogel 2007). In jüngster Zeit häufen sich Forderungen, Verhandlungslösungen auch auf nationaler Ebene zuzulassen (vgl. Teichmann 2008, S. 797f.) um, dem Beispiel der Europäischen Aktiengesellschaft (Societas Europaea - SE) folgend, auf die Herausforderungen zu reagieren, die durch europäischen Wettbewerb für die nationale Mitbestimmung entstünden. ${ }^{1}$

In der Tat war in der jahrzehntelangen Debatte um die Schaffung der SE die Frage um die Ausgestaltung der Arbeitnehmerbeteiligung stets kontrovers. Das Projekt geriet zu einer Art „Seifenoper“, mit „ständig neuen Entwürfen in einer sich verschiebenden Landschaft von Mitgliedsländern und politisch-ökonomischen Konjunkturen“ (Sorge 2006, S. 251). Erst der 1997 vorgelegte Bericht der von der Kommission eingesetzten sogenannten Davignon-Gruppe formulierte einen Kompromiss: Der SE wurde keines der bestehenden nationalen Systeme zugrunde gelegt, sondern eine verpflichtende Arbeitnehmerbeteiligung, die eine in ,uneingeschränkter Freiheit ausgehandelte Lösung“ und auf die „jeweiligen kulturellen Unterschiede zugeschnitten" sein sollte. Eine solche „flexible“ Lösung erhielt Vorrang vor Auffangregelungen, die nur im Falle eines Scheiterns freier Verhandlungen greifen sollten (Sachverständigengruppe 1997). Der Gipfel von Nizza beschloss Ende 2001 die „Verordnung des Rates über das Statut der Europäischen Gesellschaft" (EG/2157/2001) (nachfolgend: SE-VO) sowie die „Richtlinie des Rates zur Ergänzung des Statuts der Europäischen Gesellschaft hinsichtlich der Beteiligung der Arbeitnehmer" (2001/86/EG) (nachfolgend: SE-RL).

Die SE stellt eine rein optionale Rechtsform dar, die zu den bestehenden nationalen Regelungen ergänzend hinzutritt. Seit Ende 2004 ist die Gründung einer SE möglich. Von Anfang an stieß die neue Option auf ein gewisses Interesse (Keller/Werner 2007); bis Ende 2008 wurde sie von ca. 300 Unternehmen im Europäischen Wirtschaftsraum (EWR) aufgegriffen und genutzt.

\subsection{UNTERSUCHUNGSGEGENSTAND}

Die gerade in letzter Zeit deutlich gestiegene Anzahl an SE wirft Fragen nach deren
Auswirkungen auf. Dieser Beitrag analysiert die Situation der SE, vor allem die verhandelte Arbeitnehmerbeteiligung, nach aktuellem Stand und zieht eine erste, empirisch fundierte Bilanz aus einer IndustrialRelations-Perspektive. Der Artikel gliedert sich wie folgt: Abschnitt 2 gibt eine quantitativ-deskriptive, generelle Bestandsaufnahme zur SE. Abschnitt 3 erläutert institutionelle Grundlagen zur verhandelten Arbeitnehmerbeteiligung, die Basis sind für das Verständnis der weiteren Abschnitte. Abschnitt 4 behandelt die Ebene des SEBetriebsrates (im Folgenden SE-BR). Die Abschnitte 5 bis 7 gehen auf ausgewählte Phänomene und Probleme ein, die aus Industrial-Relations-Perspektive sowie aus

\footnotetext{
Die Autoren danken Dr. Norbert Kluge, Dr. Roland Köstler, Dr. Gudrun Linne sowie den beiden anonymen Gutachtern für zahlreiche Gespräche und kritische Anregungen.

1 Der Arbeitskreis „Unternehmerische Mitbestimmung " (2009) hat dazu jüngst einen Gesetzentwurf vorgelegt, um, so die Autoren, einer "Flucht aus der $A G$ " in die SE zu begegnen.
}

Berndt Keller, Prof. Dr., lehrt Arbeits- und Sozialpolitik an der Universität Konstanz. Arbeitsschwerpunkte: Arbeitspolitik des öffentlichen Sektors, Folgeprobleme der europäischen Integration, Flexicurity, Atypische Beschäftigung, Zusammenschlüsse von Gewerkschaften.

e-mail: Berndt.Karl.Keller@uni-konstanz.de Frank Werner, Universität Konstanz, Dokto rand im Promotionskolleg "Arbeitnehmerinteressen und Mitbestimmung in einem Europäischen Sozialmodell" der Hans Böckler-Stiftung (www.sozialmodell.eu) e-mail: Frank.Werner@uni-konstanz.de 
deutscher Sicht relevant sind. Dabei behandelt Abschnitt 5 diejenigen SE, die von der Möglichkeit der freien Wahl der Organstruktur Gebrauch machen, d.h. die vom dualistischen Vorstands- und Aufsichtsratssystem ins monistische Board-System wechseln. Abschnitt 6 fokussiert auf die Unternehmen, die die SE als Vehikel zur Einschränkung bzw. zum Einfrieren von Mitbestimmung nutzen. Abschnitt 7 thematisiert das spezielle Phänomen aktivierter Vorrats-SE. Abschließend werden mögliche Auswirkungen auf die tradierte deutsche Mitbestimmung reflektiert (Abschnitt 8).

Der Beitrag greift bestimmte, relevante und interessante Aspekte im Zusammenhang mit der verhandelten Arbeitnehmerbeteiligung in der SE heraus. ${ }^{2}$ Die empirische Basis der Untersuchung bilden Daten der "European Company Database“ (www.worker-participation.eu $)^{3}$ des Europäischen Gewerkschaftsinstituts (EGI), die durch eigene Recherchen, Interviews und Dokumentenanalysen ergänzt wurden.

\section{2 \\ Quantitativ-deskriptive Bestandsaufnahme}

Die Errichtung einer SE erfordert - zumindest rein rechtlich gemäß Statut - ein grenzüberschreitendes Element und ein Stammkapital von mindestens $120.000 €$. Die Gründung kann auf vier Arten erfolgen (Art. 2 SE-VO), wobei bereits aus deren Form bestimmte Implikationen für Inhalte und Ausgestaltung der Arbeitnehmerbeteiligung resultieren können (Köstler 2008). Eine SE kann gegründet werden

- durch Verschmelzung von Aktiengesellschaften aus zwei Mitgliedstaaten,

- durch Gründung einer Holding-SE von Unternehmen (z. B. AG und $\mathrm{GmbH}$ ) aus zwei Mitgliedstaaten,

\begin{tabular}{lc}
\hline $\begin{array}{l}\text { Tabelle 1: Gründungen normaler } \\
\text { SE im Zeitverlauf }\end{array}$ \\
\hline Jahr & $\begin{array}{c}\text { Zahl gegründeter } \\
\text { normaler SE }\end{array}$ \\
\hline 2004 & 2 \\
2005 & 4 \\
2006 & 11 \\
2007 & 26 \\
2008 & 30 \\
\hline
\end{tabular}

Quelle: European Company

Database 2008 und
Recherchen der Autoren. WSI MITTEILUNGEN
- durch Gründung einer Tochter-SE von Gesellschaften oder juristischen Personen öffentlichen oder privaten Rechts aus zwei Mitgliedstaaten (oder durch Gründung einer Tochter-SE durch eine bereits bestehende SE),

- durch Umwandlung einer bestehenden Aktiengesellschaft in eine SE, wenn diese seit mindestens zwei Jahren eine Tochtergesellschaft in einem anderen Mitgliedstaat hat.

\subsection{DER EMPIRISCHE REGELFALL: ATYPISCHE SE}

Eine quantitative Bestandsaufnahme nach mehr als vier Jahren Erfahrung zeigt folgende Verteilung: Am 31.12.2008 waren 307 SE in die Handelsregister der Mitgliedstaaten des EWR eingetragen. Nicht bei allen registrierten SE handelt es sich um Unternehmen, die sowohl wirtschaftliche Aktivitäten aufweisen als auch Arbeitnehmer beschäftigen. Ohnehin sind mehrere Subtypen zu unterscheiden, die z. T. unerwartete Charakteristika aufweisen:

- Leere SE verfügen über wirtschaftliche Aktivitäten, z. B. halten sie Vermögen, beschäftigen jedoch keine Arbeitnehmer;

- Vorrats-SE weisen weder das eine noch das andere Merkmal auf;

- von sogenannten UFO-SE ist nur ihre Existenz durch die Eintragung im Handelsregister bekannt. Mit großer Wahrscheinlichkeit handelt es sich ebenfalls um leere bzw. Vorrats-SE.

234 der 307 registrierten SE, also gut drei Viertel, gehören zu einem dieser drei Typen. Unsere ursprüngliche Vermutung war, dass sie aus Industrial-Relations-Perspektive nicht weiter interessant seien, da es ohne Arbeitnehmer auch keine Beteiligung geben könne (Keller/Werner 2007). Mittlerweile wurde aber eine zweistellige Zahl von Vorrats-SE aktiviert, sodass nun auch Arbeitnehmer betroffen sind. Wie später ausgeführt (Abschnitt 7), werden mit der Aktivierung von Vorrats-SE bzw. mit der Errichtung einer SE über den Weg einer Vorrats-SE oft nicht nur Ziele wie die Beschleunigung des Gründungsprozesses, sondern auch mitbestimmungsrelevante Absichten verfolgt. Zum Teil wird über die Aktivierung einer Vorrats-SE wohl bewusst der Prozess der Verhandlungen zur Arbeitnehmerbeteiligung umgangen.

\subsection{DER EMPIRISCHE AUSNAHME- FALL: NORMALE SE}

Die folgende Analyse beruht im Gegensatz zu den drei genannten Subtypen auf normalen SE, also Unternehmen mit wirtschaftlichen Aktivitäten und Arbeitnehmern. Nur 73 der 307 im EWR registrierten SE, und damit ungefähr ein Viertel, sind normale Gesellschaften. ${ }^{4}$ In dieser Zahl sind 20 aktivierte Vorrats-SE enthalten. Im Zeitverlauf ist ein deutlicher Anstieg der Gründungen zu beobachten (Tabelle 1).

Die Verteilung nach Unternehmensgröße und -branche weist nach wie vor keine eindeutigen Tendenzen auf. Die Größe schwankt beträchtlich: von niedrigen einstelligen Arbeitnehmerzahlen bis hin $\mathrm{zu}$ sechsstelligen. Einige SE mit Sitz in Deutschland liegen nahe an mitbestimmungsrelevanten Schwellenwerten (Abschnitt 6). Normale SE finden sich sowohl im Produktions- als auch im Dienstleistungssektor. Sektorspezifische Merkmale spielen als Motive für eine SE-Gründung keine wesentliche Rolle (Übersicht 1$).^{5}$

Bei der Verteilung nach dem Sitzland verfestigt sich der Trend, dass die meisten normalen SE nur in einer begrenzten Anzahl von Ländern registriert werden. Lediglich in knapp der Hälfte der EWR-Mitgliedstaaten (14 von 30) bestehen überhaupt normale SE. Deutschland als Sitz-

2 Für eine systematische Analyse der Verhandlungen über alle Fälle verweisen wir auf Keller/Werner 2008a und 2008b.

3 In diese Datenbank, welche die umfassendste und genaueste Datenquelle zu bestehenden SE darstellt, fließen Informationen von Berichterstattern aus allen EWR-Mitgliedstaaten ein. Selbst die Kommission nutzt diese Datenbank. Dagegen sind die im Amtsblatt der Europäischen Union veröffentlichten Eintragungen unvollständig und teilweise fehlerhaft. Nach Art. 14 SE-VO muss eigentlich jede SE-Gründung dort veröffentlicht werden, was offenbar einigen nationalen Registerbehörden nicht bekannt ist.

4 Zusätzlich zu diesen 73 bereits registrierten sind mehr als 20 weitere normale Unternehmen bekannt (vgl. European Company Database 2008), die die Errichtung einer SE nicht nur prüfen, sondern bereits entsprechende Schritte eingeleitet haben.

5 Bei einer wenig differenzierten Betrachtung, die nicht nur normale, sondern alle, auch arbeitnehmerlose SE berücksichtigt, überwiegt dagegen schon aufgrund der Tatsache, dass leere SE oft Holding- bzw. Investmentgesellschaften sind, der Sektor der Finanzdienstleistungen (Eidenmüller et al. 2008a). In der Solarbranche sind, gemessen an ihrer Größe, jüngst überproportional viele Gründungen zu verzeichnen. 


\begin{tabular}{|c|c|c|c|c|c|}
\hline \multicolumn{6}{|c|}{ Übersicht 1: "Normale" SE } \\
\hline Unternehmen (Name) & Sitzland & Sektor & $\begin{array}{l}\text { Arbeitnehmer- } \\
\text { zahl }\end{array}$ & Organstruktur & $\begin{array}{l}\text { Datum der } \\
\text { Eintragung }\end{array}$ \\
\hline ABN AMRO Nordic Securities SE & Schweden & Finanzdienstleistungen & 322 & Monistisch & 30.09 .05 \\
\hline Allianz Investment Management SE & Deutschland & Finanzdienstleistungen & ca. $350-500$ & Monistisch & 12.06 .07 \\
\hline Allianz SE & Deutschland & Finanzdienstleistungen & 133.846 & Dualistisch & 13.10 .06 \\
\hline ASIC SE & Deutschland & IT & 1.429 & Dualistisch & 31.10 .08 \\
\hline BASF SE & Deutschland & Chemie & 65.590 & Dualistisch & 14.01 .08 \\
\hline Bitzer SE & Deutschland & Metall & & Monistisch & 14.04 .08 \\
\hline BVE Holding SE & Deutschland & Finanzdienstleistungen & 2 & Monistisch & 19.06 .08 \\
\hline Carthago Value Invest SE & Deutschland & Finanzdienstleistungen & 5 & Dualistisch & 15.02 .06 \\
\hline Catalis SE & Niederlande & IT & 444 & Monistisch & 25.01 .08 \\
\hline Colostrum Gesundheitsprodukte SE & Deutschland & Handel & & Dualistisch & 27.10 .08 \\
\hline \multicolumn{5}{|l|}{ Conrad Holding SE } & 18.08 .06 \\
\hline Convergence CT SE & Deutschland & Medical Engineering & 3 & Monistisch & 31.01 .06 \\
\hline Conwert Immobilien Invest SE & Österreich & Immobilien & 79 & Monistisch & 14.12 .07 \\
\hline Demonta Handel SE & Tschechische Republik & Metall & ca. 30 & & 01.06 .07 \\
\hline Donata Holding SE & Deutschland & Kosmetische Industrie & 3.922 & Monistisch & 21.03 .06 \\
\hline DVB Bank SE & Deutschland & Finanzdienstleistungen & 437 & Dualistisch & 01.10 .08 \\
\hline Elcoteq SE & $\begin{array}{l}\text { Luxemburg } \\
\text { (früher Finnland) }\end{array}$ & Metall & 7.450 & Monistisch & 01.10 .05 \\
\hline Equens SE & Niederlande & Finanzdienstleistungen & & Dualistisch & 17.07 .08 \\
\hline Eurotunnel SE & Belgien & Logistik & & Monistisch & 04.04 .06 \\
\hline Fresenius SE & Deutschland & Chemie and Hospitals & 45.777 & Dualistisch & 13.07.07 \\
\hline Galleria di Brennero & Österreich & Bau & 33 & Dualistisch & 17.12. 04 \\
\hline \multicolumn{6}{|l|}{ Brennerbasistunnel BBT SE } \\
\hline Graphisoft SE & $\begin{array}{l}\text { Ungarn } \\
\text { (früher Niederlande) }\end{array}$ & IT & 253 & Monistisch & 27.07 .05 \\
\hline Gütermann SE & Deutschland & Textilindustrie & ca. 800 & Dualistisch & 13.08 .08 \\
\hline Hager SE & Deutschland & Metall & ca. 7.600 & Dualistisch & 15.06 .07 \\
\hline HAWE Hydraulik SE & Deutschland & Metall & ca. 1.800 & Dualistisch & 05.08 .08 \\
\hline HITEUROPE SE & Deutschland & Logistik & & Monistisch & 06.11 .08 \\
\hline Huber Group Holding SE & Deutschland & Metall & ca. 100 & Dualistisch & 08.04 .08 \\
\hline I. M. Skaugen SE & Norwegen & Logistik & ca. 1.500 & Monistisch & 20.12 .07 \\
\hline Informa Deutschland SE & Deutschland & Medien & ca. 250 & Monistisch & 07.02 .08 \\
\hline Interseroh SE & Deutschland & Metall & 1.729 & Dualistisch & 24.09 .08 \\
\hline Istrokapital SE & Zypern & Finanzdienstleistungen & & & 01.02 .08 \\
\hline Joh. A. Benckiser SE & $\begin{array}{l}\text { Österreich } \\
\text { (früher Deutschland) }\end{array}$ & Finanzholding & & Monistisch & 10.04 .07 \\
\hline Klöckner \& Co. SE & Deutschland & Metall & 7.377 & Dualistisch & 08.08 .08 \\
\hline Knauf Interfer SE & Deutschland & Metall/Dienstleistungen & 1.667 & Dualistisch & 27.06 .08 \\
\hline Luxury \& Sport Cars SE & Lettland & Handel & & Dualistisch & 06.06 .07 \\
\hline Lyreco CE SE & Slowakei & Handel & ca. 30 & Monistisch & 08.10 .05 \\
\hline MAN Diesel SE & Deutschland & Metall & 6.682 & Dualistisch & 31.08 .06 \\
\hline Max Bögl International SE & Deutschland & Bau & & Dualistisch & 09.11 .07 \\
\hline Mensch und Maschine SE & Deutschland & IT & 350 & Monistisch & 07.12 .06 \\
\hline NEW YORKER SE & Deutschland & Handel & & Monistisch & 13.11 .08 \\
\hline Nh-Trans SE & Tschechische Republik & Logistik & & & 31.07 .07 \\
\hline NordiTube Technologies SE & $\begin{array}{l}\text { Deutschland } \\
\text { (früher Schweden) }\end{array}$ & Metall & ca. 38 & Monistisch & 30.05 .08 \\
\hline Odfjell SE & Norwegen & Logistik & ca. 3.500 & Monistisch & 23.07 .07 \\
\hline Odfjell Terminals SE & Norwegen & Logistik & 860 & Monistisch & 23.07 .07 \\
\hline Olivenbauer SE & Deutschland & Gastronomie & ca. 40 & Monistisch & 19.09 .06 \\
\hline Omnia Holding SE & Tschechische Republik & Immobilien & ca. 5 & & 02.08 .07 \\
\hline Orchestra Service SE & Deutschland & IT & 60 & Monistisch & 15.06 .07 \\
\hline PCC SE & Deutschland & Chemie & 3.756 & Monistisch & 05.02 .07 \\
\hline Plansee SE & Österreich & Metall & 1.422 & Monistisch & 11.02 .06 \\
\hline Porsche Automobil Holding SE & Deutschland & Automobilindustire & 11.571 & Dualistisch & 13.11 .07 \\
\hline Prosafe SE & Norwegen & Ölindustrie & 55 & Monistisch & 02.02 .07 \\
\hline Q-Cells SE & Deutschland & Solarindustrie & ca. 2.500 & Dualistisch & 23.10 .08 \\
\hline REPHORMS SE & Deutschland & Bildung & & Monistisch & 23.12 .08 \\
\hline Riga RE SE & Lettland & Finanzdienstleistungen & 31 & Dualistisch & 11.08 .06 \\
\hline RKW SE & Deutschland & Chemie & 2.221 & Dualistisch & 08.10 .08 \\
\hline Sapodo SE & Deutschland & IT & 7 & Monistisch & 13.11 .07 \\
\hline SCOR Global Life SE & Frankreich & Finanzdienstleistungen & 801 & Monistisch & 25.07 .07 \\
\hline SCOR Global P\&C SE & Frankreich & Finanzdienstleistungen & 801 & Monistisch & 03.08 .07 \\
\hline SCOR SE & Frankreich & Finanzdienstleistungen & 801 & Monistisch & 25.06 .07 \\
\hline SE Sampo Life Insurance & Estland & Finanzdienstleistungen & 110 & Dualistisch & 12.01 .07 \\
\hline Seesam Life Insurance SE & Estland & Finanzdienstleistungen & ca. 200 & Dualistisch & 29.10 .07 \\
\hline Sevic Systems SE & Deutschland & Elektronik & ca. 100 & Monistisch & 15.03 .07 \\
\hline SGS SandersGeoScience SE & Deutschland & Electronik/IT & & Monistisch & 04.11 .08 \\
\hline Solon SE & Deutschland & Solarindustrie & Ca. 850 & Dualistisch & 02.12 .08 \\
\hline SpiritON MEDIA Holding SE & Deutschland & Medien & & Monistisch & 21.11 .07 \\
\hline Strabag Bauholding SE & Österreich & Bau & 32.682 & Dualistisch & 12.10 .04 \\
\hline Surteco SE & Deutschland & Papier/Kunststoff & 2.109 & Dualistisch & 20.11 .07 \\
\hline transGourmet Holding SE & Deutschland & Handel & ca. 21.000 & Monistisch & 20.11 .08 \\
\hline Vapiano SE & Deutschland & Gastronomie & ca. 100 & Dualistisch & 10.09 .08 \\
\hline Wackler Holding SE & Deutschland & Gebäudedienstleistungen & & Monistisch & 03.11 .08 \\
\hline Wiener Privatbank SE & Österreich & Finanzdienstleistungen & & Monistisch & 23.08 .08 \\
\hline WIKA International SE & Deutschland & Metall & ca. 1.850 & Dualistisch & 01.12 .08 \\
\hline Wilo SE & Deutschland & Metall & 1.871 & Dualistisch & 24.07 .08 \\
\hline
\end{tabular}

Quelle: European Company Database 2008 und eigene Recherchen. 


\begin{tabular}{|c|c|c|c|c|c|}
\hline Land & $\begin{array}{c}\text { Zahl normaler SE } \\
\text { zum 31.12.08 }\end{array}$ & $\begin{array}{c}\text { Zahl UFO-SE } \\
\text { zum 31.12.08 }\end{array}$ & $\begin{array}{l}\text { Zahl leerer SE } \\
\text { zum 31.12.08 }\end{array}$ & $\begin{array}{c}\text { Zahl Vorrats-SE } \\
\text { zum 31.12.08 }\end{array}$ & Gesamt \\
\hline Tschechische Republik & 3 & 48 & 3 & 60 & 114 \\
\hline Deutschland & 45 & 5 & 7 & 20 & 77 \\
\hline Niederlande & 2 & 17 & 9 & 0 & 28 \\
\hline Luxemburg & 1 & 10 & 1 & 1 & 13 \\
\hline Österreich & 6 & 2 & 2 & 1 & 11 \\
\hline Vereinigtes Königreich & 0 & 7 & 3 & 1 & 11 \\
\hline Belgien & 1 & 6 & 0 & 1 & 8 \\
\hline Frankreich & 3 & 5 & 0 & 0 & 8 \\
\hline Zypern & 1 & 5 & 1 & 0 & 7 \\
\hline Norwegen & 4 & 3 & 0 & 0 & 7 \\
\hline Schweden & 1 & 0 & 0 & 3 & 4 \\
\hline Slowakische Republik & 1 & 2 & 1 & 0 & 4 \\
\hline Estland & 2 & 1 & 0 & 0 & 3 \\
\hline Lettland & 2 & 1 & 0 & 0 & 3 \\
\hline Ungarn & 1 & 0 & 2 & 0 & 3 \\
\hline Dänemark & 0 & 2 & 0 & 0 & 2 \\
\hline Liechtenstein & 0 & 2 & 0 & 0 & 2 \\
\hline Irland & 0 & 1 & 0 & 0 & 1 \\
\hline Spanien & 0 & 0 & 1 & 0 & 1 \\
\hline Gesamt & 73 & 117 & 30 & 87 & 307 \\
\hline
\end{tabular}

land überwiegt eindeutig (Tabelle 2). Diese empirische Verteilung kontrastiert mit Behauptungen der deutschen Arbeitgeberund Wirtschaftsverbände, die wiederholt argumentieren, dass deutsche Unternehmen in einer sich zunehmend internationalisierenden Wirtschaft wegen der vergleichsweise weitreichenden Form der Mitbestimmung bei der SE-Gründung benachteiligt seien. Dies würde zu einer Verschlechterung der Wettbewerbsbedingungen führen und es deutschen Unternehmen erschweren, Partner z. B. für Fusionen oder die Gründung einer SE zu finden (BDA/BDI 2004). Tatsächlich gilt das Gegenteil: Vor allem deutsche Unternehmen nutzen diese Option, um nationale Vorgaben zur Mitbestimmung einzuschränken oder einzufrieren (vgl. Abschnitt 6).

\section{3}

Zum Verständnis der empirischen Analysen sind einige Anmerkungen zur Arbeitnehmerbeteiligung notwendig. Die Grundsatzentscheidung für die SE fällen die Leitungsorgane ohne direkte Interventionsmöglichkeit der Arbeitnehmervertreter. Die Unternehmensleitung stellt einen sogenannten Gründungsplan auf, der unter anderem über die Form der SE entscheidet.
Anschließend muss die Unternehmensleitung Verhandlungen zur Beteiligung der Arbeitnehmer einleiten mit dem Ziel, eine Vereinbarung abzuschließen. Ohne Verhandlungen ist eine Eintragung der SE in das Handelsregister grundsätzlich nicht möglich (Art. 12 SE-VO). Insofern wird einer einseitigen Entscheidung des Managements vorgebeugt.

Für Umfang und Inhalte der Arbeitnehmerbeteiligung macht die Richtlinie, wie erwähnt, keine detaillierten inhaltlich-materiellen Vorgaben. Alle Einzelheiten sind frei verhandelbar mit grundsätzlich offenem Ausgang. Die Vereinbarung soll mindestens Verabredungen enthalten zur Unterrichtung und Anhörung der Arbeitnehmer in einem Vertretungsorgan oder SE-BR, sie kann auch Fragen der Unternehmensmitbestimmung regeln, z. B. die „Zahl der Mitglieder des Verwaltungs- oder Aufsichtsorgans der SE, welche die Arbeitnehmer wählen [...] und die Rechte dieser Mitglieder" (Art. 4 SE-RL). Es wird also in der Regel über Beteiligung auf zwei Ebenen verhandelt.

In den Verhandlungen repräsentiert ein „besonderes Verhandlungsgremium“ (im Folgenden BVG) die Arbeitnehmer; die Unternehmensleitung vertritt die Arbeitgeberseite. Die Mitglieder des BVG werden, vereinfachend ausgedrückt, proportional zur Zahl der betroffenen Arbeitnehmer in den Mitgliedstaaten gewählt (Art. 3 SE-RL) (Fulton 2006). In Unternehmen, in denen bereits ein EBR bestand, spiegelt die Zusam- mensetzung des BVG überwiegend diejenige des EBR wider. ${ }^{6}$ Diese Konstruktion erleichtert die Kooperation zwischen den einzelnen BVG-Mitgliedern sowie den Austausch von Informationen, da auf eine bereits institutionalisierte Form der Zusammenarbeit zurückgegriffen werden kann. Die Tatsache, dass erfahrene Arbeitnehmervertreter beteiligt sind, wirkt positiv auf die inhaltliche Kompetenz des BVG. Seine Mitglieder sind überwiegend in der zuständigen Gewerkschaft organisiert, vor allem, wenn sie aus größeren Ländern bzw. Produktionsstätten kommen. ${ }^{7}$

Beide Seiten können externe Experten zu den Verhandlungen hinzuziehen (Art. 3 (5) SE-RL). Die Kosten für Sachverständige des BVG sind vom Unternehmen zu tragen. Ihre Anzahl ist in den meisten Umsetzungsgesetzen auf einen Sachverständigen beschränkt (EWCs Bulletin 2006). Die zuständige nationale Gewerkschaft hat oft eine besondere Rolle bei der Unterstützung der Arbeit des BVG; meistens erfolgt eine Rückkopplung zu den europäischen Gewerkschaftsförderationen.

Des Weiteren können gerade Vertreter der europäischen Ebene zu einer Balance unternehmensspezifischer und eher genereller Arbeitnehmerinteressen beitragen. Die Gewerkschaftsföderationen haben eine weitere Aufgabe: Wie bereits im Falle der EBR erstellen sie für die Verhandlungen Richtlinien und Handreichungen (EMF 2003). Die SE ist eine weitere Herausforderung, die die Fähigkeiten der Gewerkschaften zu supranationaler Koordinierung fordert - sowie Ressourcen verlangt und bindet.

Die eigentlichen Verhandlungen zwischen BVG und Unternehmensleitung dauern sechs Monate und können bis zu einem Jahr verlängert werden (Art. 5 SE-RL). Drei Verhandlungsergebnisse sind grundsätzlich möglich (Köstler 2008):

(1) Der „Regelfall“ ist der Abschluss einer Vereinbarung (Art. 4 SE-RL). Sie erfolgt im Hinblick auf Inhalte in „unbeschadet [er...]

\footnotetext{
6 Das BVG wird immer neu errichtet. Häufig gewählt werden jedoch Arbeitnehmer, die Mitglied eines bereits existierenden EBR sind; ein EBR selbst kann nie als BVG fungieren.

7 Die gesonderte Vertretung der leitenden Angestellten ist nur in der BRD rechtlich-institutionell abgesichert. Es gibt nur wenige Ausnahmen, in denen einzelne nationale Belegschaften leitende Angestellte ins BVG entsenden. Die Gründe liegen eher in der Persönlichkeit als in der Position.
} 
Autonomie der Parteien“; jedoch gilt im Falle der Gründung durch Umwandlung, dass alle Komponenten und das Ausmaß der Arbeitnehmerbeteiligung Bestandsschutz haben müssen (Vorher-NachherPrinzip). Eine Vereinbarung regelt im Normalfall beide Ebenen. Bei der Unterrichtung und Anhörung der Arbeitnehmer werden Regelungen getroffen zum Vertretungsorgan/SE-BR (z. B. Zusammensetzung, Befugnisse und Rechte zur Information, Konsultation und gegebenenfalls Mitentscheidung, Sitzungshäufigkeit, Arbeitsausstattung). Bei der Arbeitnehmerbeteiligung in den Leitungsorganen wird z. B. über die Zahl der Mitglieder im Aufsichtsrat oder im Board verhandelt.

(2) Wenn beide Parteien bis zum Ende des Verhandlungszeitraums keine Vereinbarung erzielen, greifen die vorgesehenen Auffangregelungen (Art. 7 (1) SE-RL).

(3) Schließlich ist eine „Nulllösung“ möglich, wenn das BVG keine Verhandlungen mit der Unternehmensleitung aufnimmt oder diese abbricht. Für die Beteiligung der Arbeitnehmer gelten dann weiterhin die nationalen Regelungen, zusätzlich findet gegebenenfalls die EBR-RL Anwendung.

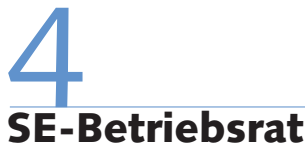

Informations- und Konsultationsrechte bzw. Vertretungsorgane zur Unterrichtung und Anhörung der Arbeitnehmer werden häufig in SE-BR realisiert - auch wenn nationale Pfadabhängigkeiten zu beobachten sind und beispielsweise SE mit Sitz in Frankreich ein „comité d'entreprise“ bilden. In Unternehmen, in denen vorher Europäische Betriebsräte (EBR) existierten, ähneln die SE-BR diesen oft im Hinblick auf Mitglieder und Strukturen. Hinsichtlich der Arbeitsteilung zwischen SE-BR und nationalen Gremien wird häufig ein Passus zu Kompetenzabgrenzungen aufgenommen (orientiert an SE-RL Anhang Teil 2a und $₫ 27$ SEBG): Der SE-BR ist für die Angelegenheiten zuständig, welche die SE selbst bzw. Arbeitnehmer in mehreren Mitgliedstaaten betreffen, während die nationalen Gremien weiterhin verantwortlich sind für alle Fragen, die lediglich nationale Arbeitnehmer bzw. einen nationalen Unternehmensteil anbelangen. Oft re- geln die Vereinbarungen, dass die nationalen Gremien bestimmte Aufgaben auf den SE-BR übertragen können, solange nationale rechtliche Anforderungen nicht berührt werden.

Die Größe des SE-BR berechnet sich im Regelfall nach der Anzahl der Arbeitnehmer in der SE bzw. nach ihrer nationalen Verteilung. Bei der Mehrzahl bestehen kleinere Steuerungsgremien für die tägliche Arbeit, die sich meist aus dem Vorsitzenden und seinen Stellvertretern zusammensetzen.

Die Rechte des SE-BR sind in allen Vereinbarungen beschränkt auf Unterrichtung und Anhörung. Verhandlungsrechte im Sinne echter Mitentscheidungsrechte, wie sie das deutsche Betriebsverfassungsgesetz (BetrVG) in bestimmten Angelegenheiten vorgibt (z. B. \$\$87,91 BetrVG), sind in keinem Fall vorgesehen. Aus Arbeitnehmersicht gibt es im Vergleich zu EBR wegen der eindeutigeren rechtlichen Voraussetzungen durchaus Verbesserungen. Diese betreffen vor allem technisch-institutionelle Fragen wie eine weitergehende Institutionalisierung der Treffen mit dem Management, bestimmte Initiativrechte oder die Einführung eines "Controlling“-Systems zur Evaluation der Zusammenarbeit beider Seiten.

Die Ausstattung mit Ressourcen bestimmt Optionen und Zwänge eines SEBR. Freistellungen spielen eine entscheidende Rolle. Bedeutsam ist auch der Schutz vor Diskriminierung durch den Arbeitgeber, wie ihn z. B. der Kündigungsschutz gewährt. Die Vereinbarungen verweisen häufig auf die nationalen Bestimmungen, die für den jeweiligen Arbeitnehmer gelten. Gerade Kündigungsschutzregelungen können für einzelne Mitglieder unterschiedlich ausfallen.

Die Mehrzahl der Vereinbarungen sieht jährlich zwei ordentliche Zusammenkünfte mit der Unternehmensleitung vor; bisweilen konnten sich die Parteien nur auf ein Treffen einigen. Außerordentliche $\mathrm{Zu}$ sammenkünfte sind möglich, wenn „essenzielle“ oder „unvorhersehbare“ Ereignisse eintreten, die die Arbeitnehmer betreffen. Vorbereitende Treffen des SE-BR sind ebenfalls vereinbart. Alle Kosten für diese Treffen trägt das Unternehmen.

In Bezug auf Fort- und Weiterbildung für die Mitglieder des SE-BR wird den Arbeitnehmervertretern in den meisten Vereinbarungen eine relativ große Autonomie gewährt. Bestimmte inhaltliche Themengebiete sind in den Vereinbarungen übli- cherweise nicht vorgegeben, die Maßnahmen müssen lediglich für die „Arbeit des SE-Betriebsrats erforderliche Kenntnisse vermitteln", wie es in einer Vereinbarung exemplarisch heißt. In den meisten Fällen versuchen die Unternehmensleitungen, Englisch als Arbeitssprache durchzusetzen, um die Kosten für Simultanübersetzungen zu reduzieren. Folglich werden die Mitglieder des SE-BR in den Vereinbarungen häufig aufgefordert, entsprechende Sprachkurse zu besuchen. Eine zwingende Regelung ist dies jedoch nicht, sodass die Unternehmensleitungen auch weiterhin akzeptieren, die Kosten für Dolmetscher zu tragen. Die Ausgaben für externe Sachverständige, die der SE-BR hinzuzieht, übernehmen gemäß der Mehrzahl der Vereinbarungen gleichfalls die Unternehmen.

\section{5 \\ SE und die freie Wahl der Organstruktur}

Die Grundsatzentscheidung zur SE-Gründung fällen, wie bereits angesprochen, die Leitungsorgane ohne direkte Interventionsmöglichkeit der Arbeitnehmervertreter. Die Unternehmensleitung stellt einen sogenannten Gründungsplan auf, der u. a. über die Form der SE entscheidet. Auch die Organstruktur wird festgelegt, wobei zwei Grundtypen von Corporate Governance infrage kommen: Zum einen eine monistische, wie sie im Vereinigten Königreich üblich ist. Das bedeutet: Ein Verwaltungsorgan, das sogenannte Board, führt das Unternehmen. Zum anderen ist eine dualistische Struktur möglich, wie sie u.a. in Deutschland üblich ist, d. h. Unternehmensleitung und -kontrolle werden in Vorstand und Aufsichtsrat separiert. ${ }^{8}$

Die SE ermöglicht erstmals einen Wechsel zwischen den Organstrukturen. In einem Staat, in dem bisher nur die dualistische Form zugelassen war, kann nunmehr auch eine monistische SE gegründet werden - und umgekehrt. Nach herrschender Meinung der juristischen Literatur kann die Entscheidung nicht Gegenstand der

\footnotetext{
Zu einer Übersicht, in welchen Ländern das nationale Recht monistische bzw. dualistische Leitungssysteme vorsieht, vgl. Kluge/Stollt 2006. Es gibt auch Länder, in denen das nationale Recht eine Wahlmöglichkeit bzw. "kombinierte Systeme" erlaubt (z. B. Frankreich, vgl. Keller 2002).
} 


\section{Übersicht 2: Normale SE mit monistischer Organstruktur}

Unternehmen (Name)

Allianz Investment Management SE

Bitzer SE

BVE Holding SE

Conrad Electronic SE and Conrad Holding SE

Convergence CT SE

Donata Holding SE

HITEUROPE SE

Informa Deutschland SE

Mensch und Maschine SE

NEW YORKER SE

Olivenbauer SE

Orchestra Service SE

PCC SE

REPHORMS SE

Sapodo SE

Sevic Systems SE

SGS SandersGeoScience SE

SpiritON MEDIA Holding SE

transGourmet Holding SE

Wackler Holding SE

NordiTube Technologies SE

Quelle: European Company Database 2008 und Recherchen der Autoren. Die Tabelle

berücksichtigt nur SEs

mit Sitz in Deutschland.

Verhandlungen zur Arbeitnehmerbeteiligung sein, selbst wenn jüngst gegenteilige Überlegungen angestellt werden (Teichmann 2008, S. 805). ${ }^{9}$

Eine Analyse der normalen SE zeigt, dass beide Formen tatsächlich vorkommen. Besonders aufschlussreich ist der Blick auf die SE mit Sitz in Deutschland: Die Mehrzahl der Unternehmen folgt dem dualistischen System - auch die großen SE mit mindestens fünfstelligen Arbeitnehmerzahlen (Übersicht 1). In den meisten Fällen spiegelt die gewählte Organstruktur die des Unternehmens vor Errichtung der SE - und damit die für das Sitzland typische - wider. Dieser Sachverhalt ist nicht überraschend, da organisationale Kontinuität bzw. Pfadabhängigkeit mehrfach nachgewiesen wurde (Villiers 2006).

Die Ergebnisse zeigen, dass es in fünf der 24 SE mit dualistischer Organstruktur paritätische Mitbestimmung gibt; ${ }^{10}$ sechs SE haben eine Drittelbeteiligung der Arbeitnehmer im Aufsichtsrat und dreizehn SE überhaupt keine Arbeitnehmerbeteiligung. Letzteres trifft nicht nur auf kleinere SE mit weniger als 500 Arbeitnehmern zu, sondern auch für SE solcher Größe, die als Unternehmen nach deutschem Recht eigentlich einen mitbestimmten Aufsichtsrat haben müssten, diesen aber vor der SEGründung nicht eingerichtet hatten (bzw. die kurz vor dieser Schwelle standen, vgl.
Abschnitt 6). ${ }^{11}$ Dieses Ausnutzen lückenhafter gesetzlicher Vorgaben scheint relativ häufig und von der Unternehmensgröße abhängig zu sein. ${ }^{12}$

Ein weiterer, bis dato unerwarteter Trend ergibt sich im Zusammenhang mit den Überwachungs- und Aufsichtsorganen. Die Grundsatzentscheidung über deren Größe treffen ausschließlich die Eigentümer in Zusammenarbeit mit dem Vorstand, d.h. vor allem die Hauptversammlung hat hier die Befugnisse. Diese Entscheidung fällt bereits mit dem Gründungsplan, also vor den Verhandlungen über Arbeitnehmerbeteiligung. ${ }^{13}$

Bei SE mit dualistischer Organstruktur und paritätisch besetztem Aufsichtsrat erfolgt bisweilen eine Verkleinerung dieses Gremiums. ${ }^{14}$ Diese Entscheidung bedeutet nicht automatisch eine Einschränkung vorher geltender Mitbestimmungsregelungen, da die formale Parität beibehalten wird: Nach wie vor entfällt eine gleiche Zahl von Mitgliedern auf Anteilseigner- wie Arbeitnehmerseite. Bei einer detaillierten Analyse fällt auf, dass diese Reduzierung der Sitze auf beiden Seiten vor allem zulasten der externen Mitglieder geht. Auf Arbeitnehmerseite sind es hauptsächlich hauptamtliche Gewerkschaftsvertreter, deren Anzahl und damit Einfluss zugunsten betriebsinterner Arbeitnehmerrepräsentanten reduziert wird. Diese veränderte Zusammensetzung kann zur Folge haben, dass die intern-unternehmensspezifische Interessenvertretung gestärkt wird zum Nachteil extern-sektoraler Interessen bzw. genereller Belange. D.h.: Der Zuschnitt der Interessenvertretung verändert auch die inhaltliche, qualitative Interessenwahrnehmung.

Außerdem ist bei der Verteilung der Organstrukturen bemerkenswert, dass immerhin 21 der 45 normalen SE sich für die monistische Variante entscheiden und damit ein anderes Modell wählen als bisher in Deutschland vorgeschrieben (Übersicht 2). In diesen SE gibt es kein mitbestimmtes Leitungsorgan, sondern maximal Informations- und Konsultationsrechte z. B. über einen SE-BR. ${ }^{15}$ Im Übrigen kommen Wechsel in die umgekehrte Richtung nicht vor, sodass die SE dualistische Systeme tendenziell aushöhlen könnte. ${ }^{16}$

Warum wählen unerwartet viele SE mit Sitz in Deutschland eine monistische Organstruktur? Der gemeinsame Nenner besteht darin, dass es sich um eigentümerzentrierte Unternehmen handelt, bei denen der (Mehrheits-)Eigentümer stark in das
Tagesgeschäft involviert ist. Eine wie auch immer geartete Kooperation mit einem Aufsichtsrat wird als unnötig angesehen, eine Teilung der Leitungsorgane würde Strukturen und Prozesse nur verlangsamen und verteuern, so die Stellungnahmen von Beteiligten. Gerade für kleinere und mittelständische SE sind - so wurde in Interviews angeführt - die Kosten eines zusätzlichen Organs ein Motiv, eine monistische Organstruktur zu wählen. Insgesamt ergeben sich für diese Gruppe andere Begründungen als für große Publikumsgesellschaften.

\section{SE als Vehikel zur Einschränkung bzw. zum Einfrieren von Mitbestimmung}

Bei den normalen SE mit Sitz in Deutschland fällt eine weitere "Gruppe“ von Unternehmen mit gemeinsamen Merkmalen

9 Grundsätzlich ist eine Beurteilung der Effizienz der verschiedenen Systeme nur schwer möglich. Nach Fällen wie Enron oder Worldcom in den USA dürfte feststehen, dass eine monistische Organstruktur einer dualistischen nicht notwendigerweise überlegen ist, wie in den 1990er Jahren wiederholt behauptet wurde (Nagel 2006).

10 Dies ist der Fall bei MAN Diesel SE, Allianz SE, Fresenius SE, Porsche Automobil Holding SE und BASF SE.

11 Dies gilt insbesondere für die Conrad Electronic SE mit über 2.000 Arbeitnehmern in Deutschland.

12 Die Auffangregelung, die sich am tatsächlich bestehenden und nicht am rechtlich vorgeschriebenen Umfang orientiert, hätte für diese SE keine Mitbestimmung vorgesehen (Vorher-NachherPrinzip: Vorher nichts - nachher nichts).

13 In der juristischen Literatur herrscht jedoch weitgehend Einigkeit, dass die von der Hauptversammlung beschlossene Satzung angepasst werden muss, falls die Verhandlungen zur Arbeitnehmerbeteiligung eine andere Größe des Aufsichtsrats ergeben.

14 Dies war der Fall bei Allianz SE, BASF SE, MAN Diesel SE. Bei der Fresenius SE wurde eine Aufsichtsratsgröße von zwölf Mitgliedern beibehalten, trotz einer Arbeitnehmerzahl, die nach deutschem Recht einen 20-köpfigen Aufsichtsrat erfordert hätte.

15 Eine Ausnahme stellt die österreichische Plansee SE dar, bei der die Arbeitnehmer zwei Mitglieder des insgesamt fünfköpfigen Boards benennen. Für die monistischen SE mit Sitz in Deutschland muss einschränkend festgehalten werden, dass es vor der Gründung in keinem Fall Mitbestimmung gab, obwohl manche Unternehmen über den relevanten Schwellenwerten lagen; auch die Auffangregelungen hätten insofern keine Mitbestimmung vorgesehen.

16 Eidenmüller et al. (2008b) sprechen in diesem Zusammenhang von der SE als einem "vehicle for legal arbitrage ${ }^{\prime \prime}$ 


\begin{tabular}{|c|c|}
\hline \multicolumn{2}{|c|}{$\begin{array}{l}\text { Übersicht 3: Normale SE nahe mitbestimmungsrelevanten Schwellen- } \\
\text { werten (500 bzw. 2.000 Arbeitnehmer) }\end{array}$} \\
\hline SE (Name) & Arbeitnehmerzahl in D \\
\hline Bitzer SE & knapp 2.000 (strittig) \\
\hline Conrad Electronic SE/Conrad Holding SE & 2.313 \\
\hline Donata Holding SE & 564 \\
\hline GfK SE & knapp 2.000 \\
\hline Gütermann SE & 480 \\
\hline Hager SE & knapp 2.000 \\
\hline HAWE Hydraulik SE & ca. 1.500 \\
\hline Interseroh SE & 1.409 \\
\hline Klöckner \& Co SE & 1.788 \\
\hline Knauf Interfer SE & 1.652 \\
\hline Mensch und Maschine Software SE & 212 \\
\hline PCC SE & 136 \\
\hline Q-Cells SE & 1.707 \\
\hline RKW SE (Rheinische Kunststoffwerke) & knapp 2.000 \\
\hline SOLON SE & 420 \\
\hline Surteco SE & knapp 2.000 \\
\hline WIKA International SE & vmtl. ca. 1.850 \\
\hline Wilo SE & 1.871 \\
\hline \multicolumn{2}{|c|}{$\begin{array}{l}\text { Die Übersicht enthält z. T. auch SE, die noch vergleichsweise weit von den Schwellenwerten entfernt sind (z. B. Mensch und } \\
\text { Maschine SE; Interseroh SE). Unsere Recherchen belegen jedoch auch für diese Fälle, dass ein Motiv für die SE-Gründung im } \\
\text { dauerhaften Einfrieren der Mitbestimmung gelegen haben dürfte. Gleichzeitig enthält die Tabelle Fälle, die mitbestimmungsrele- } \\
\text { vante Schwellenwerte bereits überschritten haben, in denen jedoch noch kein mitbestimmter (bzw. kein paritätisch mitbestimmter) } \\
\text { Aufsichtsrat implementiert war. Auch in den Reihen geplanter SE finden sich Fälle, in denen das Einfrieren von Mitbestimmung ein } \\
\text { (zentrales) Motiv sein dürfte. }\end{array}$} \\
\hline \multicolumn{2}{|c|}{$\begin{array}{l}\text { Quelle: European Company Database } 2008 \text { und Recherchen der Autoren. Die Übersicht } \\
\text { berücksichtigt nur SE mit Sitz in Deutschland. }\end{array}$} \\
\hline
\end{tabular}

auf. Die SE ist besonders für solche Unternehmen attraktiv, die für Regelungen der Mitbestimmung relevante Schwellenwerte erreichen. Einige Unternehmen, die sich der Schwelle von 2.000 Arbeitnehmern nähern und damit in den Geltungsbereich des 1976er-Mitbestimmungsgesetzes gelangen würden, versuchen, durch Umwandlung in eine SE den bisherigen Status einzufrieren. Ähnliches gilt für Unternehmen, die den Schwellenwert von $500 \mathrm{Ar}$ beitnehmern erzielen, womit sie in den Geltungsbereich des Drittelbeteiligungsgesetzes von 2004 fallen würden (Übersicht 1 und 3).

Wiederum handelt es sich hier vor allem um Unternehmen, deren wirtschaftliche Entscheidungen ein (Mehrheits-)Inhaber trifft, der stark ins Tagesgeschäft involviert ist. Entweder wechseln sie direkt ins monistische System (vgl. Abschnitt 5) oder sie schreiben eine geringere Arbeitnehmerbeteiligung im Aufsichtsrat fest, als dies nach den Gesetzen für eine deutsche AG vorgeschrieben wäre. Diesen Unternehmen gelingt es durch die SE-Gründung, striktere nationale Formen der Mitbestimmung zu verhindern. Gleichwohl sollte der Begriff „Mitbestimmungsflucht“ mit Vorsicht verwendet werden, da solche strikteren nationalen Formen in diesen Unternehmen vormals gar nicht eingeführt waren. Folg- lich wäre „präventive Mitbestimmungsflucht" ein angemessenerer Begriff.

Die Gründung einer SE ist eine neue, vergleichsweise einfach $\mathrm{zu}$ handhabende Option, um den Status quo der Mitbestimmung einzufrieren. Früher mussten Unternehmen vergleichsweise komplizierte, damit beratungs- und kostenintensive, und zugleich rechtlich unsichere Mittel verwenden, um bestehenden Mitbestimmungsregelungen zu entgehen. Dies geschah z. B. über einen Komplementär nach ausländischem Recht (Sick 2008). Mit der SE existiert nun eine zusätzliche, kostengünstigere und rechtlich abgesicherte Alternative (Götze et al. 2009).

Dieses Phänomen des Status-quo-Erhalts der Mitbestimmung (auf niedrigem Niveau) überrascht durchaus, da es in Anbetracht der jahrzehntelangen Diskussion nicht der ursprünglichen politischen Absicht der Richtlinie entspricht, in der SE die Mitbestimmung zu sichern. Die aktuelle Verteilung der SE zeigt ein eindeutig nichtintendiertes Resultat, das eine hohe Attraktivität für andere Unternehmen haben und damit zur weiteren Verbreitung der SE in Deutschland beitragen könnte. Auch Vorrats-SE werden häufig mit dem Argument zum Verkauf angeboten, dass sie Möglichkeiten zur Einfrierung von Mitbestimmung bieten (vgl. Abschnitt 7).

\section{7 Arbeitnehmerbeteiligung in aktivierten Vorrats-SE}

20 der normalen SE mit Sitz in Deutschland (Übersicht 1 und 4) resultieren aus der Aktivierung von Vorrats-SE. Aus juristischer Perspektive (Blanke 2005) war zunächst zweifelhaft, ob solche SE überhaupt registriert werden dürfen; Voraussetzung für die Eintragung ins Handelsregister sind eigentlich, wie erwähnt, abgeschlossene Verhandlungen über die Beteiligung der Arbeitnehmer (Art. 12 SE-VO). Die HansBöckler-Stiftung versuchte, entsprechende Vorrats-SE wieder aus den Handelsregistern löschen zu lassen; die Verfahren scheiterten, da die betroffenen Unternehmen das Argument durchsetzten, dass Verhandlungen über die Beteiligung in SE ohne Arbeitnehmer schlechterdings nicht möglich seien.

Die entscheidende Frage lautet, was nach Verkauf und Aktivierung eines solchen Firmenmantels passiert. Juristische Stellungnahmen vertreten die Auffassung, dass bei der Aktivierung einer Vorrats-SEalso bei Aufnahme eines operativen Geschäfts mit Arbeitnehmern - das Recht der wirtschaftlichen Neugründung gilt und Verhandlungen zur Arbeitnehmerbeteiligung stattfinden müssen (Casper/Schäfer 2007; Oetker 2008; Bayer 2008; Köstler 2008). In einigen Fällen, in denen die Gründer keine rechtlichen Möglichkeiten für Anfechtungen aufkommen lassen wollen, werden Verhandlungen geführt; deren Ergebnisse unterscheiden sich nicht fundamental von denen in neu errichteten SE. ${ }^{17}$

In weiteren Fällen finden die Verhandlungen jedoch erst im Nachhinein, also nach der Aktivierung, bzw. gar nicht statt. Die Frage ist, ob bei Aktivierung einer Vorrats-SE eine Verpflichtung zu Verhandlungen besteht, es also zu deutlichen strukturellen Änderungen im Sinne der SE-RL kommt. Es wird letztlich auf eine gerichtliche Klärung ankommen. Solche Klagen müssten die (nationalen) Betriebsräte anstrengen, die es in manchen Fällen gibt, bzw. die zuständigen Gewerkschaften.

\footnotetext{
17 So z. B. bei der Donata Holding SE oder der ASIC
} SE. 


\section{Ausblick: Auswirkungen auf die deutsche Mitbestimmung}

Dieser Beitrag kann aufgrund der nach wie vor begrenzten, wenn auch steigenden Zahl normaler SE (noch) keine endgültigen Schlüsse ziehen hinsichtlich der Auswirkungen der SE-Gründungen sowie der geschilderten - nicht-intendierten - Ergebnisse auf die industriellen Beziehungen in den Mitgliedstaaten im Allgemeinen und in Deutschland im Besonderen. Die direkten Auswirkungen der SE auf die deutsche Mitbestimmung sind (noch) gering. Die Folgen einzelner skizzierter Veränderungen, z. B. der Verkleinerung des Aufsichtsrats (vgl. Abschnitt 5), lassen sich erst in einigen Jahren beurteilen, wenn praktische Erfahrungen vorliegen. Bei anderen Beobachtungen, z. B. der präventiven Mitbestimmungsflucht (vgl. Abschnitt 6), bleibt abzuwarten, ob sich aktuelle Trends verstetigen.

Dennoch wollen wir einige Vermutungen anstellen:

Zum einen fällt auf, dass, wie einleitend erwähnt, in jüngster Zeit wiederum Forderungen nach Veränderungen der deutschen Unternehmensmitbestimmung erhoben werden; sie stellt nach wie vor ein "contested terrain" dar, obwohl es lange Jahre den Anschein hatte, als sei sie von allen Akteuren akzeptiert. Verschiedene Interessengruppen (BDA/BDI 2004; Donges et al. 2007) verlangen mit Verweis auf den angeblich durch die SE entstehenden Druck die Einführung von Verhandlungen, die "maßgeschneiderte" unternehmensspezifische Lösungen ermöglichen sollen. Dabei wird das Argument angeführt, dass sich dieses „flexible Arrangement" bei der SE bewährt habe (Arbeitskreis 2009). Auch mit Blick auf die in der deutschen Unternehmensmitbestimmung strikt festgelegte Organstruktur kann die Debatte um Wahloptionen zwischen monistischen und dualistischen Systemen, wie es sie in der SE gibt, wieder auf die politische Agenda treten, wenn die entsprechenden politischen Mehrheiten zustande kommen. Auf mittelbzw. langfristige Sicht könnte der Verweis

\begin{tabular}{ll}
\hline Übersicht 4: Aus aktivierten Vorrats-SE resultierende normale SE \\
\hline Namen der bestehenden SE & $\begin{array}{l}\text { Name der Vorrats-SE, aus der die bestehende SE } \\
\text { resultiert }\end{array}$ \\
\hline Allianz Investment Management SE & Atrium Zweite Europäische VV SE \\
ASIC SE & Atrium Siebzehnte Europäische VV SE \\
Bitzer SE & Atrium Elfte Europäische VV SE \\
BVE Holding SE & Atrium Dreizehnte Europäische VV SE \\
Colostrum Gesundheitsprodukte SE & Sarpedon 2007/01 Vermögensverwaltungs SE \\
Convergence CT SE & Atrium Erste Europäische VV SE \\
Donata Holding SE & Atrium Fünfte Europäische VV SE \\
ENRO Energie SE & Yella SE \\
Informa Deutschland SE & Atrium Zehnte Europäische VV SE \\
Max Boegl International SE & Sarpedon 2006/01 SE \\
NEW YORKER SE & Blitz 08-851 SE \\
Olivenbauer SE & Atrium Achte Europäische VV SE \\
Orchestra Service SE & ProJura 0407 SE \\
REPHORMS SE & Blitz 08-852 SE \\
Sapodo SE & unitedcon SE \\
SpiritON MEDIA Holding SE & PRO-JURA 0307 SE \\
transGourmet Holding SE & Blitz F08-acht-sechs-zwei SE \\
Vapiano SE & Blitz F07-zwei-fünfundvierzig SE \\
Wackler Holding SE & Blitz F07-zwei-siebenundvierzig SE \\
WIKA International SE & Atrium Vierte Europäische VV SE \\
\hline Es gibt noch eine Reihe weiterer Vorrats-SE, die aktiviert wurden. Aus diesen resultierte jedoch eine leere SE. \\
Quelle: European Company Database 2008 und eigene Recherchen. Die Tabelle berück- \\
sichtigt nur SE mit Sitz in Deutschland. \\
\hline
\end{tabular}

auf die Option der SE-Gründung - und die möglichen, geschilderten Phänomene durchaus zum Drohpotenzial der Arbeitgeberseite werden und besonders bei kleinen und mittelständischen Unternehmen Schule machen.

Zum anderen: Arbeitnehmervertretern aus EWR-Mitgliedstaaten mit ausschließlich freiwilliger Beteiligungstradition (van het Kaar 2005) bietet die SE zum ersten Mal die Gelegenheit, unternehmerische Entscheidungsprozesse auf Konzernebene mitzugestalten. In verschiedenen Fällen sind Arbeitnehmervertreter aus anderen Mitgliedstaaten in die Aufsichtsräte deutscher SE eingezogen. Dennoch ist es übertrieben, hierin bereits einen „Export" von Mitbestimmung in Länder mit schwächeren Beteiligungsrechten zu sehen.

Darüber hinaus bietet die SE solchen Unternehmen, die striktere nationale Formen von Mitbestimmung vermeiden wollen, eine legale, vergleichsweise einfach zu folgen nach wie vor der tradierten Mitbestimmung zumindest in ihren Grundzügen. Neue, eventuell auch schnell wachsende Unternehmen haben mit der SE die Möglichkeit, „ihre“ spezifische Form der Corporate Governance und das von handhabende Exit-Option. Die großen SE ihnen gewünschte Maß an Arbeitnehmerbeteiligung mit der SE vergleichsweise einfach - und, falls Beteiligungsvereinbarungen unbefristet gelten, auch dauerhaft $\mathrm{zu}$ verwirklichen. ${ }^{18}$ Darüber hinaus könnten Entwicklungen in diese Richtungen durch weitere europäische Rechtsformen (wie die Europäische Privatgesellschaft oder die Europäische Genossenschaft) bzw. durch grenzüberschreitende Verschmelzungen nach der 10. Gesellschaftsrechtlichen Richtlinie verstärkt werden.

\footnotetext{
18 Einige Gemeinsamkeiten, aber auch Unterschiede betreffen die Modalitäten bezüglich Laufzeit und Kündigungsmöglichkeiten der ersten Beteiligungsvereinbarung. In den meisten Fällen wurde eine relativ lange Laufzeit vereinbart, von vier bis zu zehn Jahren. In manchen Fällen können Teile der Vereinbarung (zum SE-BR bzw. zur Mitbestimmung in den Leitungsorganen) auch getrennt gekündigt werden. Andere Vereinbarungen sehen eine Kündigungsmöglichkeit dagegen nur in Gänze vor. Die Kündigungsfristen divergieren zwischen sechs Monaten und einem Jahr. Bei "strukturellen Änderungen“ ist schon per Gesetz eine Pflicht zu Neuverhandlungen vorgesehen. Eine Definition von "strukturellen Änderungen “ wurde seitens des Gesetzgebers jedoch versäumt, sodass die Auslegung strittig ist.
} 


\section{LITERATUR}

Arbeitskreis "Unternehmerische Mitbestimmung“ (2009): Entwurf einer Regelung zur Mitbestimmungsvereinbarung sowie zur Größe des mitbestimmten Aufsichtsrats, in: Zeitschrift für Wirtschaftsrecht (ZIP) 19, S. 885-899

Bayer, W. (2008): Gründung, in: Lutter, M./Hommelhoff, P. (Hrsg.): SE-Kommentar. SE-VO - SEAG - SEBG - Steuerrecht, Köln, S. 196-372 Bundesvereinigung der Deutschen Arbeitgeberverbände (BDA)/Bundesverband der Deutschen Industrie (BDI) (2004): Bericht der Kommission Mitbestimmung. Mitbestimmung modernisieren, Berlin Bertelsmann-Stiftung/Hans-Böckler-Stiftung (Hrsg.) (1998): Mitbestimmung und neue Unternehmenskulturen - Bilanzen und Perspektiven. Bericht der Kommission Mitbestimmung, Gütersloh

Biedenkopf, K./Streeck, W./Wissmann, H. (2006): Bericht der wissenschaftlichen Mitglieder der Kommission zur Modernisierung der deutschen Mitbestimmung, Berlin

Blanke, T. (2005): „Vorrats-SE“ ohne Arbeitnehmerbeteiligung, Edition der Hans-Böckler-Stiftung 161, Düsseldorf

Casper, M./Schäfer, C. (2007): Die Vorrats-SE - Zulässigkeit und wirtschaftliche Neugründung, in: Zeitschrift für Wirtschaftsrecht 14, S. 653662

Donges, J./Eekhoff, J./Franz, W./Fuest, C./Möschel, W./Neumann, M. (2007): Unternehmensmitbestimmung ohne Zwang, Berlin Eidenmüller, H./Engert, A./Hornuf, L. (2008a): Die Societas Europaea: Empirische Bestandsaufnahme und Entwicklungslinien einer neuen Rechtsform, in: Die Aktiengesellschaft 20, S. 721-730

Eidenmüller, H./Hornuf, L./Engert, A. (2008b): Incorporating under European Law: The Societas Europaea as a Vehicle for Legal Arbitrage, http://papers.ssrn.com/sol3/papers.cfm?abstract_id=1316430

Europäischer Metallgewerkschaftsbund (EMF) (2003): The European Company - SE, EMF guidelines, Brüssel

European Company Database (2008): http://ecdb.worker-participation. eu/

EWCs Bulletin (2006): European Company Statute update, in: European Works Council Bulletin 9-10, S. 12-14

Fulton, L. (2006): The forgotten resource: corporate governance and employee board-level representation, the situation in France, the Niederlande, Schweden and the UK. Report for Hans-Böckler-Foundation, London

Götze, C./Winzer, T./Arnold, C. (2009): Unternehmerische Mitbestimmung - Gestaltungsoptionen und Vermeidungsstrategien, in: Zeitschrift für Wirtschaftsrecht (ZIP) 6, S. 245-254

Keller, B. (2002): The European Company Statute: Employee involvement - and beyond, in: Industrial Relations Journal 5, S. 423-444

Keller, B./Werner, F. (2007): Arbeitnehmerbeteiligung in der Europäischen Aktiengesellschaft (SE) - Eine empirische Analyse der ersten Fälle, in: WSI-Mitteilungen 11, S. 604-612
Keller, B./Werner, F. (2008a): Negotiated Forms of Worker Involvement in the European Company (SE) - First Empirical Evidence and Conclusions, in: Management Revue 4, S. 291-306

Keller, B./Werner, F. (2008b): The establishment of the European Company (SE) - The first cases from an industrial relations perspective, European Journal of Industrial Relations 2, S. 153-175

Kluge, N./Stollt, M. (Hrsg.) (2006): The European company - Prospects for worker board-level participation in the enlarged EU, Brüssel

Köstler, R. (2008): Die Europäische Aktiengesellschaft, Reihe "Arbeitshilfen für Aufsichtsräte" 6 , Düsseldorf

Nagel, B. (2006): Der Konflikt zwischen der deutschen und angelsächsischen Corporate Governance und die Europäische Gesellschaft (SE),

in: Brandes, W./Eger, T., Kraft, M. (Hrsg.): Wirtschaftswissenschaften zwischen Markt, Norm und Moral. Festschrift für Peter Weise, Kassel, S. 337-359

Oetker, H. (2008): SE-Beteiligungsgesetz, in: Lutter, M./Hommelhoff, P. (Hrsg.): SE-Kommentar. SE-VO - SEAG - SEBG - Steuerrecht, Köln, S. 835-1067

Renaud, S. (2007): Dynamic Efficiency of Supervisory Board Codetermination in Germany, in: Labour 4, S. 689-713

Sachverständigengruppe European Systems of Worker Involvement (1997): Abschlußbericht, Brüssel

Seyboth, M./Thannisch, R. (2008): Zukunftsfaktor Mitbestimmung, in: WSI-Mitteilungen 9, S. 519-522

Sick, S. (2008): Mitbestimmungsrelevante Unternehmen mit ausländischen/kombiniert ausländischen Rechtsformen, Düsseldorf

Sorge, A. (2006): Mitbestimmung für die Europäische Aktiengesellschaft: Nützliche Lehren aus mehr als dreißig Jahren Seifenoper, in: Beckert, J./ Ebbinghaus, B./Hassel, A./Manow, P. (Hrsg.): Transformationen des Kapitalismus. Festschrift für Wolfgang Streeck, Frankfurt/New York,

S. $249-271$

Teichmann, C. (2008): Gestaltungsfreiheit in Mitbestimmungsvereinbarungen, in: Die Aktiengesellschaft 22, S. 797-808

Van het Kaar, R. (2005): Company Law and workers' interest, in: Transfer 2, S. 179-188

Villiers, C. (2006): The Directive on employee involvement in the European Company: Its role in European corporate governance and industrial relations, in: International Journal of Comparative Labour Law and Industrial Relations 2, S. 183-211

Vitols, S. (2006): Ökonomische Auswirkungen der paritätischen Mitbestimmung: Eine ökonometrische Analyse, Berlin

Vogel, S. (2007): Social partners divided over issues of co-determination at company level, http://www.eurofound.europa.eu/eiro/2006/11/ articles/de0611039i.htm 